\title{
NONLINEAR ELLIPTIC BOUNDARY VALUE PROBLEMS. II $\left({ }^{1}\right)$ \\ BY
}

FELIX E. BROWDER

In a preceding paper on nonlinear elliptic boundary value problems [7], the writer established an existence theorem for variational solutions of nonlinear elliptic boundary value problems for systems of the form

$$
A u=\sum_{|\alpha| \leqq m} D^{\alpha} A_{\alpha}\left(x, u, \cdots, D^{m} u\right)
$$

with $A_{\alpha}$ having at most polynomial growth. This theorem was derived from an abstract theorem concerning the solvability of a class of nonlinear functional equations in reflexive Banach spaces. Our result in [7] extended and generalized earlier results anonunced by M. I. Vishik [20], [21], [22] and obtained by more concrete-analytic arguments. Very recently Vishik has published in [23] a detailed account of his methods and obtained more precise results than those announced in his Notes listed above. The one feature of the results of [23] which goes beyond the framework of the methods given in [7] (and one on which Vishik has laid great emphasis) is that the monotonicity or strong ellipticity hypotheses imposed on the system $A$ involve essentially only the variation of the $A_{\alpha}$ with respect to $D^{m} u$ and not with respect to the lower-order derivatives of $u$.

It is our object in the present discussion to give an extension of our methods which allows us to obtain results under weaker hypotheses of this type. As in [7], our approach is based on a general theorem on nonlinear functional equations in Banach spaces.

In $\S 1$, we formulate our main results on the solvability of nonlinear elliptic boundary value problems and the corresponding abstract theorem. In $\S 2$, we prove the abstract theorem. In $\S 3$, we prove our main theorem on the existence of solutions of boundary value problems. In $\$ 4$, we consider extensions and specializations of this theorem. In $\$ 5$, we turn back to the abstract theory and analyze the general method applied in [7] and here in the general context of locally convex linear spaces.

1. Let $\Omega$ be a bounded and smoothly bounded open subset of $R^{n}$. The general

Received by the editors October 30, 1963.

( $\left.{ }^{1}\right)$ The preparation of this paper was partially supported by the National Science Foundation under NSF Grant 19751 and by the Army Research Office (Durham) under Grant DA-ARO(D)31-124-G455. 
point of $R^{n}$ will be denoted by $x=\left(x_{1}, \cdots, x_{n}\right)$, while $\int f(x) d x$ will denote integration with respect to Lebesgue $n$-measure on $\Omega$. We set $D_{j}=i^{-1} \partial / \partial x$, for $1 \leqq j \leqq n$, and for each $n$-tuple $\alpha=\left(\alpha_{1}, \ldots, \alpha_{n}\right)$ of non-negative integers we set

$$
D^{\alpha}=\prod_{j=1}^{n} D_{j}^{\alpha_{j}},|\alpha|=\sum_{j=1}^{n} \alpha_{j}
$$

By functions $u$ on $\Omega$, we shall mean $s$-vector functions $u=\left(u_{1}, \cdots, u_{s}\right)$ for a fixed positive integer $s$, where each $u_{k}$ is a complex-valued function on $\Omega$. We set $D^{\alpha} u=\left(D^{\alpha} u_{1}, \cdots, D^{\alpha} u_{s}\right)$.

Let $m$ be a positive integer, $p$ a real number with $1<p<+\infty$. We define

$$
W^{m, p}(\Omega)=\left\{u \mid u \in L^{p}(\Omega), D^{\alpha} u \in L^{p}(\Omega) \text { for }|\alpha| \leqq m\right\} .
$$

(All derivatives $D^{\alpha} u$ will be taken in the sense of the theory of distributions.)

$W^{m, p}(\Omega)$ is a reflexive separable Banach space with respect to the norm

$$
\|u\|_{m, p}=\left\{\sum_{|\alpha| \leqq m} \int_{\Omega}\left|D^{\alpha} u\right|^{p} d x\right\}^{1 / p} .
$$

We denote by $C_{c}^{\infty}(\Omega)$ the family of infinitely differentiable functions with compact support in $\Omega$. We consider $C_{c}^{\infty}(\Omega)$ as a subset of $W^{m, p}(\Omega)$. Let

$$
\langle u, v\rangle=\sum_{k=1}^{s} \int u_{k}(x) \bar{v}_{k}(x) d x
$$

be the natural pairing between $u$ in $L^{p}(\Omega)$ and $v$ in $L^{q}(\Omega)$, with $q=p(p-1)^{-1}$.

We consider the system of differential operators

$$
A u=\sum_{|\alpha| \leqq m} D^{\alpha} A_{\alpha}\left(x, u, \cdots, D^{m} u\right)
$$

where for each $\alpha, A_{\alpha}$ is an $s$-vector function of $x$ in $\Omega$, the value of the function $u$ at $x$, and the values of all the derivatives $D^{\alpha} u$ at $x$ for $|\alpha| \leqq m$.

We assume the following smoothness and growth conditions on the coefficients $A_{\alpha}$ :

Assumption I. The functions $A_{\alpha}$ are measurable in $x$ on $\Omega$ and continuous in $\left(u, \cdots, D^{m} u\right)$. There exists a real number $p>1$ and a continuous function $g(r)$ of the real variable $r$ such that if

then

$$
\zeta=\left\{\zeta_{\alpha} ;|\alpha| \leqq m\right\}
$$

$$
\left|A_{\alpha}(x, \zeta)\right| \leqq g\left(\sum_{|\gamma|<m-n / p}\left|\zeta_{\gamma}\right|\right) \cdot\left\{1+\sum_{|\beta|=m}\left|\zeta_{\beta}\right|^{p-1}+\sum_{m-n / p \leqq|\beta| \leqq m-1}\left|\zeta_{\beta}\right|^{(p-1)+c_{\beta-}}\right\}
$$

where $c_{\beta \alpha}$ satisfies 


$$
0 \leqq c_{\beta \alpha}<r_{\beta} \operatorname{Min}\left\{\frac{p-1}{p}+\frac{m-|\alpha|}{n}, 1\right\}-(p-1)
$$

and $r_{\beta}$ is any exponent such that

$$
r_{\beta}^{-1} \geqq p^{-1}-n^{-1}(m-|\beta|) .
$$

To define a variational boundary value problem for the system $A$, we assume given a closed subspace $V$ of $W^{m, p}(\Omega)$ with $C_{c}^{\infty}(\Omega) \subset V$.

Corresponding to the representation (1.1) for $A$, we may define the nonlinear Dirichlet form $a(u, v)$ for each pair $u$ and $v$ in $W^{m, p}(\Omega)$ by

$$
a(u, v)=\sum_{|\alpha| \leqq m}\left\langle A_{x}\left(x, u, \cdots, D^{m} u\right), D^{\alpha} v\right\rangle .
$$

From the Sobolev Imbedding Theorem (e.g. Lemma 5 of [2]), it follows that if $u$ lies in $W^{m, p}(\Omega)$, then for $|\gamma|<m-n / p, D^{\gamma} u$ lies in $C(\bar{\Omega})$. For $|\gamma| \geqq m-n / p$, $D^{\gamma} u$ lies in $L^{r_{\gamma}}(\Omega)$ where $r_{\gamma}$ is any finite exponent such that

$$
r_{\gamma}^{-1} \geqq p^{-1}-n^{-1}(m-|\gamma|) .
$$

Moreover the norms of the maps $u \rightarrow D^{\gamma} u$ from $W^{m, p}(\Omega)$ to the spaces $C(\bar{\Omega})$ and $L^{r_{\gamma}}(\Omega)$ are bounded. Applying Assumption I and Hölder's inequality, it follows that $a(u, v)$ is well defined for all $u$ and $v$ in $W^{m, p}(\Omega)$ and satisfies an inequality of the form

$$
|a(u, v)| \leqq g_{1}\left(\|u\|_{m, p}\right)\|v\|_{m, p}
$$

where $g_{1}(r)$ is a function of the real variable $r$ depending on the function $g$ of Assumption I.

If $V^{*}$ is the conjugate space of $V$, we may now define the variational boundary value problem corresponding to the pair $(A, V)$ by:

DEFINITION. Let $f$ be an element of $V^{*},(f, v)$ its value on $v$ in $V$. Then $u$ is said to be a solution of the variational boundary value problem for $A u=f$ satisfying the null boundary conditions corresponding to the space $V$ if

(a) $u \in V$, and

(b) $a(u, v)=(f, v)$, for all $v$ in $V$.

In order to give a precise formulation of the hypotheses under which we shall prove the existence of variational solutions for our boundary problem, we shall write the nonlinear Dirichlet form $a(u, v)$ in another notation which separates its dependence on the lower order derivatives of $u$ from its dependence on the $m$ th derivatives $D^{m} u$.

DeFinition. Let

$$
a(u ; v, w)=\sum_{|\alpha| \leqq m}\left\langle A_{\alpha}\left(x, u, \ldots, D^{m-1} u, D^{m} v\right), D^{\alpha} w\right\rangle .
$$


It follows from Assumption I that there exists $r$ with $r^{-1}>p^{-1}-n^{-1}$ such that $a(u ; v, w)$ is well defined for all $u$ in $W^{m-1, r}(\Omega)$, and all $v$ and $w$ in $W^{m, p}(\Omega)$. By the Sobolev Imbedding Theorem, $W^{m, p}(\Omega)$ is contained in $W^{m-1, r}(\Omega)$ and the imbedding map is compact. Moreover

$$
|a(u ; v, w)| \leqq g_{1}\left(\|u\|_{m-1}, r\right) g_{2}\left(\|v\|_{m, p}\right)\|w\|_{m, p} .
$$

THEOREM 1. Let $A$ be a system of differential operators of the form (1.1) satisfying Assumption I for a given value of $p>1$. Let $V$ be a closed subspace of $W^{m, p}(\Omega)$ such that $C_{c}^{\infty}(\Omega) \subset V$. Let $r$ be a real number as above with $r^{-1}>p^{-1}-n^{-1}$ such that $a(u ; v, w)$ is well defined for $u \in W^{m-1, r}(\Omega), w$ and $v$ in $W^{m, p}(\Omega)$ and inequality (1.6) holds. Suppose that all of the following conditions are satisfied:

(a) For each positive integer $N$, there exists a continuous function $C_{N}(t)$ with $\lim C_{N}(t)=+\infty$ as $t \rightarrow+\infty$ such that for all $u$ in $V$ with $\|u\|_{m-1, r} \leqq N$, we have

$$
\operatorname{Re}\{a(u ; v, v-w)-a(u ; w, v-w)\} \geqq C_{N}\left(\|v-w\|_{m, p}\right)\|v-w\|_{m, p}
$$

for all $v$ and $w$ in $V$.

(b) There exists a continuous function $c(t)$ on $R^{1}$ with $c(t) \rightarrow+\infty$ as $t \rightarrow+\infty$ such that for all constants $k \geqq 1$,

$$
\operatorname{Re} a(u ; k u, u) \geqq c\left(\|u\|_{m, p}\right)\|u\|_{m, p}
$$

for all $u$ in $V$.

Then for every $f$ in $V^{*}$, there exists a solution $u$ in $V$ of the $V$-variational boundary value problem for $A u=f($ i.e. such that $a(u, v)=(f, v)$ for all $v$ in $V)$.

We shall derive Theorem 1 from the following abstract theorem concerning nonlinear operators in Banach spaces.

THEOREM 2. Let $X$ be a separable reflexive Banach space, $X^{*}$ its conjugate space (i.e. the space of bounded conjugate-linear functionals on $X)$. For $w \in X^{*}$, $u \in X$, let $(w, u)$ be the value of $w$ at $u$. Let $Y$ be a second Banach space such that the elements of $X$ are identified with a linear subset of $Y$ and the injection mapping is a compact linear map of $X$ into $Y$.

Let $G$ be a (not necessarily linear) mapping of $Y \times X$ into $X^{*}$ and for $u$ in $Y$, let $G_{u}$ be the mapping of $X$ into $X^{*}$ defined by $G_{n}(v)=G(u, v)$. Suppose that all of the following conditions are satisfied:

(a) For each positive integer $N$, there exists a continuous function $C_{N}(r)$ on $R^{1}$ with $\lim _{r \rightarrow \infty} C_{N}(r)=+\infty$ and $C_{N}(r)>0$ for $r>0$ such that

$$
\operatorname{Re}\left(G_{u} v-G_{u} w, v-w\right) \geqq C_{N}\left(\|v-w\|_{X}\right)\|v-w\|_{X}
$$

for $u$ in $Y$ with $\|u\|_{\gamma} \leqq N$ and for all $v$ and $w$ in $X$.

(b) There exists a continuous real-valued $c(r)$ on $R^{1}$ with $c(r) \rightarrow+\infty$ as $r \rightarrow+\infty$ such that for every $k \geqq 1$, 


$$
\operatorname{Re}\left(G_{u}(k u), u\right) \geqq c\left(\|u\|_{X}\right)\|u\|_{X}
$$

for all $u$ in $X$.

(c) For each $u$ in $Y, G_{u}$ is a demi-continuous mapping from $X$ to $X^{*}$ (i.e. continuous from the strong topology in $X$ to the weak topology of $\left.X^{*}\right)$. For each fixed $v$ in $X$, the mapping

$$
u \rightarrow G_{u} v
$$

is a strongly continuous mapping from $Y$ to $X^{*}$.

Then the mapping $F$ of $X$ into $X^{*}$ given by $F u=G_{u} u$ maps $X$ onto $X^{*}$, i.e. for every $w$ in $X^{*}$, there exists $u$ in $X$ such that $G(u, u)=w$.

2. Let $X$ be a separable reflexive Banach space, $X^{*}$ its conjugate space (i.e. $X^{*}$ is the space of bounded conjugate-linear functionals on $X$ ). For $w$ in $X^{*}, u$ in $X$, we denote the value of $w$ at $u$ by $(w, u)$. Thus $(w, u)$ is linear in $w$, conjugate-linear in $u$.

Let $G$ be a (not necessarily linear) mapping from $X$ into $X^{*}$.

DEFINITION. $G$ is said to be demi-continuous if it is continuous from the strong topology of $X$ to the weak topology of $X^{*}$.

Proposition 1. Let $G$ be a demi-continuous mapping of $X$ into $X^{*}$ such that

(a) For all $u$ and $v$ of $X$,

$$
\operatorname{Re}(G u-G v, u-v) \geqq 0 .
$$

(b) There exists a continuous real-valued function $c(r)$ on $R^{1}$ with $c(r) \rightarrow+\infty$ as $r \rightarrow+\infty$ such that

$$
\operatorname{Re}(G u, u) \geqq c(\|u\|)\|u\| .
$$

Then $G$ maps $X$ onto $X^{*}$.

Proof of Proposition 1. This is a special case of Theorem 2 of [7]. Since more general results are proved in $\$ 5$ below, we omit the proof here.

Proposition 2. Let $G$ be a demi-continuous mapping of $X$ into $X^{*}$ such that there exists a continuous function $c(r)$ on $R^{1}$ with $c(r)>0$ for $r>0$ and $\lim c(r)=+\infty$ as $r \rightarrow \infty$ such that

$$
\operatorname{Re}(G u-G v, u-v) \geqq c(\|u-v\|)\|u-v\|
$$

for $u$ and $v$ in $X$. Then:

(a) $G$ maps $X$ onto $X^{*}$.

(b) $G$ is one-to-one, $G^{-1}$ is defined on all of $X^{*}$ and maps bounded sets of $X^{*}$ into bounded sets of $X$.

(c) $G^{-1}$ is strongly continuous from $X^{*}$ to $X$. There exists a real-valued 
monotone nondecreasing function $h(r)$ on $R^{1}$ such that $h(0)=0, h$ is continuous from the right, and

$$
\left\|G^{-1} w-G^{-1} w_{1}\right\| \leqq h\left(\left\|w-w_{1}\right\|\right)
$$

for all $w$ and $w_{1}$ in $X^{*}$. The function $h$ depends only on the function $c(r)$ of inequality (2.1).

Proof of Proposition 2. From the inequality (2.1) and the fact that $c(r)>0$ for $r>0$, it follows that $G$ is one-to-one. Hence $G^{-1}$ is defined on the range of $G$.

For each pair $u$ and $v$ in $X$, we know from (2.1) that

$$
\operatorname{Re}(G u-G v, u-v) \geqq 0 .
$$

Moreover,

$$
\operatorname{Re}(G u, u)=\operatorname{Re}(G u-G(0), u)+(G(0), u) .
$$

Hence

$$
\begin{aligned}
\operatorname{Re}(G u, u) & \geqq c(\|u\|)\|u\|-\|G(0)\| \cdot\|u\| \\
& \geqq\{c(\|u\|)-\|G(0)\|\}\|u\| .
\end{aligned}
$$

Since $c(r)-\|G(0)\| \rightarrow+\infty$ as $r \rightarrow+\infty$, we may apply Proposition 1 and conclude that $G$ maps $X$ onto $X^{*}$, i.e. the range of $G$ is all of $X^{*}$.

For each $R \geqq 0$, let

$$
h(R)=\sup \{r \mid c(r) \leqq R\} .
$$

Then $h(R)<+\infty$ for every $R, h(0)=0$, and $h(R)$ is monotone nondecreasing in $R$. Obviously $h(R)$ depends only on the function $c(r)$. Finally, $h(R)$ is continuous from the right. Indeed suppose otherwise. Then there exists a sequence $R_{j} \rightarrow R$ such that $h\left(R_{j}\right)=r_{j} \geqq h(R)+\varepsilon$. Then $c\left(r_{j}\right)=R_{j}$ and extracting a convergent subsequence from the bounded sequence $r_{j}$, we will have $r_{j} \rightarrow r \geqq h(R)+\varepsilon$, and $c\left(r_{j}\right) \rightarrow R=c(r)$. This contradicts the definition of $h(R)$. Thus $h(R)$ is continuous from the right. From the inequality (2.1), we know that

If $u \neq v$, we have

$$
c(\|u-v\|)\|u-v\| \leqq\|G u-G v\| \cdot\|u-v\| .
$$

$$
c(\|u-v\|) \leqq\|G u-G v\|
$$

Hence

$$
\|u-v\| \leqq h(\|G u-G v\|) .
$$

Setting $u=G^{-1} w, v=G^{-1} w_{1}$, we obtain

$$
\left\|G^{-1} w-G^{-1} w_{1}\right\| \leqq h\left(\left\|w-w_{1}\right\|\right) .
$$


Suppose $w_{j} \rightarrow w_{1}$ strongly in $X^{*}$. Then $\left\|w_{j}-w_{1}\right\| \rightarrow 0$. Since $h(0)=0$ and $h(R)$ is continuous from the right, $h\left(\left\|w_{j}-w_{1}\right\|\right) \rightarrow 0$. Hence $\left\|G^{-1} w_{j}-G^{-1} w_{1}\right\| \rightarrow 0$ as $j \rightarrow+\infty$, i.e. $G^{-1} w_{j} \rightarrow G^{-1} w_{1}$ strongly in $X$. Hence $G^{-1}$ is continuous from $X^{*}$ to $X$.

Finally if $\|w\| \leqq M$, then

$$
\left\|G^{-1} w-G^{-1}(0)\right\| \leqq h(\|w\|) \leqq h(M) .
$$

Thus

$$
\left\|G^{-1} w\right\| \leqq h(M)+\left\|G^{-1}(0)\right\|
$$

and $G^{-1}$ maps bounded sets of $X^{*}$ into bounded sets of $X$.

Q.E.D.

Proof of Theorem 2. Let $u$ be an element of $Y$. Then the corresponding mapping $G_{u}$ of $X$ into $X^{*}$ satisfies the hypotheses of Proposition 2 with a function $C_{N}(r)$ which is uniform over all $u$ with $\|u\|_{Y} \leqq N$.

By Proposition 2, $G_{u}$ is a one-to-one mapping of $X$ onto $X^{*}$ with a continuous inverse $G_{u}^{-1}$. This inverse satisfies an inequality of the form

$$
\left\|G_{u}^{-1} w-G_{u}^{-1} w_{1}\right\|_{X} \leqq k_{N}\left(\left\|w-w_{1}\right\|_{X^{*}}\right)
$$

for all $u$ in $Y$ with $\|u\|_{Y} \leqq N$.

Let $w$ be a fixed element of $X^{*}$. We wish to find a soluon $u$ it of the equation

$$
G_{u}(u)=w .
$$

For each $v$ in $Y$, let $T$ be the mapping of $Y$ into $Y$ given by

$$
T v=G_{v}^{-1}(w) .
$$

Then $u$ will be a solution of (2.4) if and only if $T u=u$, i.e. if $u$ is a fixed point of $T$ in $Y$.

We shall show the existence of such a fixed point $u$ of $T$ by applying the LeraySchauder theory of the degree for compact displacements in the Banach space $Y$ [14]. To do this it suffices to show the following:

(i) $T$ is compact.

(ii) There exists $M>0$ such that for $0 \leqq t \leqq 1$, the mapping $T_{t}$ with $T_{t} v=t T v$ has no fixed points $u$ with $\|u\|_{Y}=M$.

Proof of (i). Let $J$ be the imbedding map of $X$ into $Y$. $J$ is a compact linear map by assumption. Let $S$ be a bounded set in $Y$. Then for all $v$ in $S$, there exists a function $k_{N}(r)$ such that

$$
\left\|G_{v}^{-1} w-G_{v}^{-1} w_{1}\right\|_{X} \leqq k_{N}\left(\left\|w-w_{1}\right\|_{X^{*}}\right) .
$$

Setting $w_{1}=0$, we see that

$$
\left\|G_{v}^{-1} w\right\|_{x} \leqq k_{0}
$$

for all $v$ in $S$. Hence $T v=G_{v}^{-1}(w)$ lies in a compact subset of $Y$, i.e. $T(S)$ is compact. 
To show that $T$ is a compact mapping, it suffices therefore to show that $T$ is continuous. Let $v$ and $v_{0}$ be elements of $Y, u=T v, u_{0}=T v_{0}$. Then

$$
G_{v} u=w, \quad G_{v_{0}} u_{0}=w,
$$

so that

$$
G_{v} u-G_{v} u_{0}=G_{v_{0}} u_{0}-G_{v} u_{0}=\left(G_{v_{0}}-G_{v}\right) u_{0} \text {. }
$$

Suppose $v_{j} \rightarrow v_{0}$. Then $\left\|v_{j}\right\|_{Y},\left\|v_{0}\right\|_{Y} \leqq N$, so that for every $j$

$$
\left\|G_{v_{j}}^{-1} f-G_{v_{j}}^{-1} f_{1}\right\|_{X} \leqq k_{N}\left(\left\|f-f_{1}\right\|_{X^{*}}\right)
$$

for all $f$ and $f_{1}$ in $X^{*}$. Setting $f=G_{v_{j}} u, f_{1}=G_{v_{j}} u_{0}$, we find that

$$
\begin{aligned}
\left\|T v_{j}-T v_{0}\right\|_{X} & =\left\|u-u_{0}\right\|_{X} \leqq k_{N}\left(\left\|G_{v_{j}} u-G_{v_{j}} u_{0}\right\|_{X^{*}}\right) \\
& \leqq k_{N}\left(\left\|\left(G_{v_{j}}-G_{v_{0}}\right) u_{0}\right\|_{X^{*}}\right) .
\end{aligned}
$$

Since $v_{j} \rightarrow v_{0}$ strongly in $Y$ and $u_{0}$ is a fixed element of $X$, we see from the hypothesis of Theorem 2

$$
\left(G_{v_{j}}-G_{v_{0}}\right) u_{0} \rightarrow 0 \text { in } X^{*}
$$

i.e.

$$
\left\|\left(G_{v_{j}}-G_{v_{0}}\right) u_{0}\right\|_{X^{*}} \rightarrow 0 .
$$

Since $k_{N}(r) \rightarrow 0$ as $r \rightarrow 0$, it follows that

$$
k_{N}\left(\left\|\left(G_{v_{j}}-G_{v_{0}}\right) u_{0}\right\|_{X^{*}}\right) \rightarrow 0
$$

so that $\left\|T v_{j}-T v_{0}\right\|_{X} \rightarrow 0$, i.e., $T v_{j} \rightarrow T v_{0}$ in $X$ and a fortiori in $Y$. Thus $T$ is continuous from $Y$ to $Y$ and the proof that $T$ is compact is complete.

Proof of (ii). Let $t$ be a real number from the interval $0<t \leqq 1$, and suppose $u$ is a fixed point of $T_{t}$ where $T_{t} u=t T u$. Then

$$
t T u=u,
$$

i.e.

$$
G_{u}^{-1}(w)=\frac{1}{t} u
$$

or

$$
G_{u}\left(\frac{1}{t} u\right)=w .
$$

If $t=0$, the only fixed point of $T_{0}$ is zero. If $t>0$, let $k=1 / t$. Then $k \geqq 1$, and we have

$$
G_{u}(k u)=w
$$

Then 


$$
\left(G_{u}(k u), u\right)=(w, u) \text {. }
$$

From hypothesis (b) of Theorem 2, we know that

$$
c\left(\|u\|_{X}\right)\|u\|_{X} \leqq \operatorname{Re}\left(G_{u}(k u), u\right) \leqq\|w\|_{X^{*}} \cdot\|u\|_{X} .
$$

Hence $c\left(\|u\|_{X}\right) \leqq\|w\|_{X^{*}}$. Since $c(r) \rightarrow+\infty$ as $r \rightarrow+\infty$, it follows that $\|u\|_{X} \leqq M$, for some $M>0$ independent of $t$. Since the injection of $X$ into $Y$ is bounded,

$$
\|u\|_{Y} \leqq M_{1}
$$

for all such fixed points $u$ and (ii) is proved.

As we remarked above, Theorem 2 follows from (i) and (ii) and the LeraySchauder theory of the degree.

Q.E.D.

3. We now turn to the proof of Theorem 1 using Theorem 2. To carry through this proof, we shall need some preliminary results with which we begin our discussion.

LEMMA 3.1. Let $N$ be a constant and let $f\left(x, u_{1}, \cdots, u_{N}\right)$ be measurable in $x$ on $\Omega$ for fixed $u=\left(u_{1}, \cdots, u_{N}\right)$ and continuous in $u$ for fixed $x$ for almost all $x$ in $\Omega$. Let $\left\{u^{(j)}\right\}$ be an infinite sequence of functions on $\Omega$ converging in measure on $\Omega$ to $u^{(0)}(x)$ (i.e. with each component $u_{r}^{(j)}(x)$ converging in measure to $\left.u_{r}^{(0)}(x)\right)$. Then $f^{(j)}(x)=f\left(x, u_{1}^{(j)}(x), \cdots, u_{N}^{(j)}(x)\right)$ converges in measure on $\Omega$ to $f\left(x, u_{1}^{(0)}(x), \cdots, u_{N}^{(0)}(x)\right)$.

Proof of Lemma 3.1. For $N=1$, this is a result of Nemytski given on pp. 29-30 of Krasnoselski [12]. The proof for $N>1$ is the same as for $N=1$. We give it here for the sake of completeness because of the relative inaccessibility of the reference.

We consider $N$-vector functions $u(x)$ on $\Omega$ and $N$-vector constants $u$. Let $\varepsilon>0$ be a fixed positive number. Suppose $u^{(j)}(x)$ converges in measure on $\Omega$ to $u^{(0)}(x)$. For each $k \geqq 1$, let

$$
G_{k}=\left\{x|x \in \Omega,| f\left(x, u^{(0)}(x)\right)-f(x, u) \mid<\varepsilon \text { if } u \in C^{N},\left|u^{(0)}(x)-u\right|<\frac{1}{k}\right\} .
$$

The $G_{k}$ are nondecreasing with $k$ and since $f(x, u)$ is continuous in $u$ for almost all $x$ in $\Omega, \Omega-\bigcup_{k} G_{k}$ has measure zero. If $\eta>0$ is given, we may therefore find an integer $k_{0}$ such that $m\left(G_{k_{0}}\right)>m(\Omega)-\eta / 2$.

Now let

$$
F_{j}=\left\{x|x \in \Omega,| u^{(0)}(x)-u^{(j)}(x) \mid<\frac{1}{k_{0}}\right\} .
$$

Since $u^{(j)}$ converges to $u^{(0)}$ in measure, we can find $N$ such that for $j>N$, $m\left(F_{j}\right)>m(\Omega)-\eta / 2$. Then $x$ in $G_{k_{0}} \cap F_{j}$ lies in the set 


$$
D_{j}=\left\{x|| f\left(x, u^{(0)}(x)\right)-f\left(x, u^{(j)}(x)\right) \mid<\varepsilon\right\}
$$

and thus

$$
\begin{aligned}
m\left(D_{j}\right) & \geqq m(\Omega)-\left\{m\left(\Omega-G_{k_{0}}\right)+m\left(\Omega-F_{j}\right)\right\} \\
& \geqq m(\Omega)-\eta .
\end{aligned}
$$

Since $m\left(D_{j}\right) \rightarrow m(\Omega)$ for fixed $\varepsilon$ as $j \rightarrow \infty, f\left(x, u^{(j)}(x)\right)$ converges in measure to $f\left(x, u^{(0)}(x)\right)$.

Q.E.D.

LEMMA 3.2. Let $f\left(x, u_{1}, \cdots, u_{N}\right)$ be continuous in $u=\left(u_{1}, \cdots, u_{N}\right)$ for almost all $x$ and measurable in $x$ for all $u$. Suppose

$$
\left.\left|f\left(x, u_{1}, \cdots, u_{N}\right)\right| \leqq c\left\{\sum_{r=1}^{N}\left|u_{r}\right|^{c_{r}}+1\right\} \quad \text { (c, } c_{r} \text { constants }\right) .
$$

Let $Z=\prod_{r=1}^{N} L^{c_{r} p}(\Omega)$ for some $p \geqq 1$. Then the mapping Tof $Z$ into $L^{p}(\Omega)$ given by

$$
T\left(u_{1}, \cdots, u_{N}\right)(x)=f\left(x, u_{1}(x), \cdots, u_{N}(x)\right)
$$

is a continuous mapping of $Z$ into $L^{p}(\Omega)$.

Proof of Lemma 3.2. (This is a generalization of a result of M. M. Vainberg for $N=1$. Cf. [12] and [18].)

Let $u(x)$ denote the vector function $\left(u_{1}(x), \cdots, u_{N}(x)\right)$. Suppose $u^{(j)}(x)$ converges strongly to $u^{(0)}(x)$ in $Z$. Then $u^{(j)}(x)$ converges to $u^{(0)}(x)$ in measure on $\Omega$. By Lemma 3.1, $f\left(x, u^{(j)}(x)\right)$ converges in measure to $f\left(x, u^{(0)}(x)\right)$ on $\Omega$. Thus given $\varepsilon>0$ and $\eta>0$, we may find $j_{0}$ depending on $\varepsilon$ and $\eta$ such that for $j \geqq j_{0}$, the set $F_{j}$ given by

$$
F_{j}=\left\{x|| f\left(x, u^{(0)}(x)\right)-f\left(x, u^{(j)}(x)\right) \mid \geqq \varepsilon\right\}
$$

has measure $m\left(F_{j}\right)<\eta$.

We have then, however,

$$
\begin{aligned}
\delta_{j} & =\int_{\Omega}\left|f\left(x, u^{(0)}(x)\right)-f\left(x, u^{(j)}(x)\right)\right|^{p} d x \leqq\left(\int_{\Omega-F_{j}}+\int_{F_{j}}\right) \cdots \\
& \leqq \varepsilon^{p} m(\Omega)+c^{p} \int_{F_{j}}\left(\sum_{r=1}^{N}\left\{\left|u_{r}(x)\right|^{c_{r}}+\left|u_{r}^{(j)}(x)\right|^{c_{r}}\right\}+1\right)^{p} d x .
\end{aligned}
$$

For the last integral, we know that

$$
\begin{aligned}
\left.\int_{F}\left(\sum_{r=1}^{N}\left|u_{r}(x)\right|^{c_{r}}+\left|u_{r}^{(j)}(x)\right|^{c_{r}}\right\}+1\right)^{p} d x \\
\quad \leqq \sup _{j \geqq 1}\left[\mathfrak{g}(2 N+1)^{p-1} \int_{F}\left(\sum_{r=1}^{N}\left\{\left|u_{r}(x)\right|^{p c_{r}}+\left|u_{r}^{(j)}(x)\right|^{p c}\right\}+1\right) d x\right]=\mu(F)
\end{aligned}
$$


where $\mu(F) \rightarrow 0$ as $m(F) \rightarrow 0$. Since

$$
\delta_{j} \leqq \varepsilon^{p} m(\Omega)+c^{p} \mu(F)
$$

we may choose $\eta$ so small that

$$
c^{p} \mu(F)<\varepsilon^{p}
$$

and obtain the inequality

$$
\delta_{j} \leqq \varepsilon^{p}(m(\Omega)+1),
$$

so that

$$
\left\|f\left(x, u^{(j)}\right)-f\left(x, u^{(0)}\right)\right\|_{L P(\Omega)} \leqq \varepsilon(m(\Omega)+1)^{1 / p}
$$

for $j \geqq j_{0}$.

Proposition 3.1. Let $A_{\alpha}(x, \zeta), \zeta=\left\{\zeta_{\alpha},|\alpha| \leqq m\right\}$, satisfy the conditions of Assumption I. Let $r$ be any exponent such that

$$
\frac{1}{r} \geqq \frac{1}{p}-\frac{1}{n}
$$

and for every $\alpha$ and for $\beta$ with $m-1 \geqq|\beta| \geqq m-n / p$

$$
\left[(p-1)+c_{\beta \alpha}\right]\left\{r^{-1}-\frac{m-1-|\beta|}{n}\right\} \leqq \operatorname{Min}\left\{\frac{p-1}{p}+\frac{m-|\alpha|}{n}, 1\right\}
$$

( $c_{\beta \alpha}$ being the exponents of Assumption I).

Then if $T_{\alpha}$ is given by

$$
T_{a}(u, v)(x)=A_{a}\left(x, u(x), \cdots, D^{m-1} u(x), D^{m} v(x)\right),
$$

then $T_{a}$ is a continuous mapping of $W^{m-1, r}(\Omega) \times W^{m, p}(\Omega)$ into $L^{p_{\alpha}}(\Omega)$ where

$$
\frac{1}{p_{\alpha}}=\operatorname{Min}\left\{\frac{p-1}{p}+\frac{m-|\alpha|}{n}, 1\right\} \text {. }
$$

Proof of Proposition 3.1. By Assumption I, $A_{a}(x, \zeta)$ is measurable in $x$ and continuous in $\zeta$. Moreover it satisfies the inequality

$$
\begin{aligned}
&\left|A_{2}(x, \zeta)\right| \leqq g\left(\sum_{|\gamma|<m-n / p}\left|\zeta_{\gamma}\right|\right) \\
& \cdot\left\{\sum_{|\beta|=m}\left|\zeta_{\beta}\right|^{p-1}+\sum_{m-n / p \leqq|\beta|<m}\left|\zeta_{\beta}\right|^{(p-1)+c_{\beta \nu}}+1\right\} .
\end{aligned}
$$

Applying Lemma 3.2, we need only to show that for $u \in W^{m-1, r}(\Omega)$ ( $r$ as above) and $v$ in $W^{m-1, p}(\Omega)$, we have

$$
\begin{array}{ll}
D^{\beta} u \in L^{p_{\alpha}\left[p-1+c_{\beta \alpha}\right],} & |\beta| \leqq m-1, \\
D^{\beta} v \in L^{p_{\alpha}(p-1)}, & |\beta|=m,
\end{array}
$$


and that the mapping $u \rightarrow D^{\beta} u$ and $v \rightarrow D^{\beta} v$ in each of these cases is a bounded linear map of $W^{m-1, r}(\Omega)$ and $W^{m, p}(\Omega)$, respectively, into the appropriate $L^{p}$ spaces.

To do this, we apply the Sobolev Imbedding Theorem (e.g. Lemma 5 of [2]) and find that for $u$ in $W^{m-1, r}(\Omega), D^{\beta} u$ lies in $W^{m-1-|\beta|, r}(\Omega)$ and hence in $L^{r \beta}(\Omega)$ where

$$
\frac{1}{r_{\beta}} \geqq \frac{1}{r}-\frac{m-1-|\beta|}{n} \text {. }
$$

It suffices for this case to show that

$$
\frac{1}{r}-\frac{m-1-|\beta|}{n} \leqq \frac{1}{(p-1)+c_{\beta \alpha}} \frac{1}{p_{\alpha}},
$$

i.e.

$$
\left[(p-1)+c_{\beta \alpha}\right]\left\{r^{-1}-n^{-1}(m-1-|\beta|)\right\} \leqq \operatorname{Min}\left\{1, \frac{p-1}{p}+\frac{m-|\alpha|}{n}\right\} .
$$

This is precisely our hypothesis on $r$.

For $|\beta|=m$, we must note that if $v \in W^{m p}(\Omega)$ then $D^{\beta} u \in L^{p}(\Omega)$. It suffices then to prove that

or

$$
p_{\alpha}(p-1) \leqq p
$$

$$
p-1 \leqq p / p_{x}
$$

However

$$
\frac{1}{p_{\alpha}}=\operatorname{Min}\left\{\frac{p-1}{p}+\frac{m-|\alpha|}{n}, 1\right\}
$$

so that

$$
\frac{p}{p_{\alpha}} \geqq(p-1)+p\left\{\frac{m-|\alpha|}{n}\right\} \geqq p-1 .
$$

The boundedness of the imbedding mappings follows from the Sobolev Imbedding Theorem.

Q.E.D.

In order to apply Theorem 2 to the proof of Theorem 1, we must transform the problem of the existence of the solution of the boundary value problem considered in Theorem 1 into the type of functional equation treated in Theorem 2.

We now let $r$ be an exponent such that

$$
\frac{1}{r}>\frac{1}{p}-\frac{1}{n}
$$

while for all $\alpha$ and all $\beta$ with $m-n|p \leqq| \beta \mid \leqq m-1$,

$$
\frac{1}{r} \leqq \frac{m-1-|\beta|}{n}+\left[(p-1)+c_{\beta a}\right]^{-1} \operatorname{Min}\left\{\frac{p-1}{p}+\frac{m-|\alpha|}{n}, 1\right\} \text {. }
$$


Let $X$ be the closed subspace $V$ of $W^{m, p}(\Omega)$ endowed with the Banach space structure induced by $W^{m, p}(\Omega)$. Let $Y$ be the closure of $V$ in $W^{m-1, r}(\Omega)$ with the norm of $W^{m-1, r}(\Omega)$.

For $u$ in $Y, v$ and $w$ in $X$, we consider the form

$$
a(u ; v, w) \text {. }
$$

This is conjugate linear in $w$ and satisfies the bounds

$$
|a(u ; v, w)| \leqq g\left(\|u\|_{Y},\|v\|_{X}\right)\|w\|_{X} .
$$

Hence there exists a unique element of $X$ which we shall denote by $G(u, v)$ that

$$
(G(u, v), w)=a(u ; v, w)
$$

for all $w$ in $X$.

We shall show that $G$ satisfies the hypotheses of Theorem 2 .

Proposition 3.2. The mapping $G$ of $X \times Y$ into $X^{*}$ defined byequation (3.4) above is continuous.

As a corollary, we have the fact that the mapping of $X$ into $X^{*}$ given by $v \rightarrow G(u, v)$ is continuous.

Proof of Proposition 3.2. To show that $G$ is continuous, it suffices to show that if $u_{j} \rightarrow u_{0}$ in $Y, v_{j} \rightarrow v_{0}$ in $X$, then

$$
\left|\left(G\left(u_{j}, v_{j}\right)-G\left(u_{0}, v_{0}\right), w\right)\right| \leqq \varepsilon_{j}\|w\|_{X}
$$

where $\varepsilon_{j} \rightarrow 0$ as $j \rightarrow+\infty$.

However,

$$
\left(G\left(u_{j}, v_{j}\right)-G\left(u_{0}, v_{0}\right), w\right)=a\left(u_{j} ; v_{j}, w\right)-a\left(u_{0} ; v_{0}, w\right)
$$

while

$$
\begin{aligned}
& a\left(u_{j} ; v_{j}, w\right)-a\left(u_{0} ; v_{0}, w\right) \\
& \quad=\sum_{|\alpha| \leqq m}\left\langle A_{\alpha}\left(x, u_{j}, \cdots, D^{m-1} u_{j}, D^{m} v_{j}\right)-A_{\alpha}\left(x, u_{0}, \cdots, D^{m-1} u_{0}, D^{m} v_{0}\right), D^{\alpha} w\right\rangle .
\end{aligned}
$$

The term indexed by $\alpha$ in the last sum has its absolute value bounded by

$$
\left\|A_{\alpha}\left(x, u_{j}, \cdots, D^{m-1} u_{j}, D^{m} v_{j}\right)-A_{\alpha}\left(x, u_{0}, \cdots, D^{m-1} u_{0}, D^{m} v_{0}\right)\right\|_{L^{p_{\alpha}}}\left\|D^{\alpha} w\right\|_{L^{r_{\alpha}}}
$$

where $1 / r_{\alpha} \geqq 1 / p-(m-|\alpha|) / n, 1 / p_{\alpha}=\operatorname{Min}\left(1-1 / r_{\alpha}, 1\right)$. By Proposition 3.1, the first norm approaches zero as $j \rightarrow \infty$. The second norm is bounded by $\|w\|_{m, p}$ by the Sobolev Imbedding Theorem. Hence

$$
\left|a\left(u_{j} ; v_{j}, w\right)-a\left(u_{0} ; v_{0}, w\right)\right| \leqq \varepsilon_{j}\|w\|_{m, p}
$$

where $\varepsilon_{j} \rightarrow 0$ as $j \rightarrow+\infty$.

Q.E.D. 
Proof of Theorem 1. We wish to apply Theorem 2 to the mapping $G$ defined above. Hypotheses (a) and (b) of Theorem 1 imply hypotheses (a) and (b) of Theorem 2 for the mapping $G$. The only point that must be checked is that the inequality of (a) of Theorem 2 for $G$ holds not only for $u$ in $V$ but also for $u$ in $Y$. However, we have for $u$ in $V$ with $\|u\|_{Y} \leqq N$,

$$
\operatorname{Re}\{(G(u, v), v-w)-(G(u, w), v-w)\} \geqq C_{N}\left(\|w-v\|_{X}\right)\|w-v\|_{X} .
$$

Both sides of the inequality are continuous in $u$ on $Y$ by Proposition 3.2. Hence if the inequality holds for all $u$ in the dense subset $V$ of $Y$, it holds for all $u$ in $Y$.

Finally hypothesis (c) on $G$ follows from the continuity of $G$ as a map of $X \times Y$ into $X^{*}$.

Q.E.D.

4. In the present section, we shall consider extensions and specializations of Theorem 1.

The extensions of Theorem 1 that we give below move in two directions. In the first place, we have decomposed the form $a(u, v)$ in two variables $u$ and $v$ into a form $a(u ; w, v)$ in three variables $u$ in $W^{m-1, r}(\Omega), w$ and $v$ in $W^{m, p}(\Omega)$ in a very particular way by replacing all the derivatives $D^{\beta} u$ with $|\beta|=m$ by $D^{\beta} w$ and leaving the lower-order derivatives alone. Under suitable hypotheses, it may be more advantageous to make a more refined choice of the occurrences of $u$ and its derivatives which are to be replaced by the corresponding terms in $w$ and $D^{\beta} w$, respectively.

The second direction of generalization concerns the generality of the boundary value problem. In the linear case, we know that new boundary problems can be treated by adding boundary terms to the Dirichlet form $a(u, v)$. If we carry through the corresponding formulation for the present case, we obtain a much wider class of boundary value problems. We simplify the application of this principle by assuming the boundary forms to be continuous with respect to the $W^{m, p}(\Omega)$ norm. This is not necessary if we introduce a new norm on $V$ involving a boundary norm with respect to which the boundary form is continuous.

As a specific representation of the two types of generalization we have just described, we combine these techniques to obtain the following result:

TheOREM 3. Let $\Omega$ be a bounded, smoothly bounded open set in $R^{n}$ with boundary $T, A$ a system of $s$ differential operators of order $2 m$ acting on $s$ vector functions $u=\left(u_{1}, \cdots, u_{s}\right)$ of the form

$$
A u=\sum_{|\alpha| \leqq m} D^{\alpha} A_{\alpha}\left(x, u, \ldots, D^{m} u\right) .
$$

Let $\zeta=\left\{\zeta_{x} ;|\alpha| \leqq m\right\}, \eta=\left\{\eta_{\beta} ;|\beta| \leqq m-1\right\}$ lie in $C^{N_{m}}, C^{N_{m}-1}$, respectively, where $N_{m}$ is the number of $\alpha$ with $|\alpha| \leqq m, N_{m-1}$ the number of $\beta$ with $|\beta| \leqq m-1$. We assume that there exist functions 


$$
E_{\alpha}(x, \eta, \zeta), \quad|\alpha| \leqq m,
$$

such that $A_{\alpha}(x, \zeta)=E_{\alpha}(x, \zeta, \zeta)$ for all $\zeta \in C^{N m}$, with $E_{\alpha}$ measurable in $x$ and continuous in $(\eta, \zeta)$. We suppose that the $E_{\alpha}$ satisfy the analogue of the bounds of Assumption I, namely:

$\left|E_{\alpha}(x, \eta, \zeta)\right|$

$$
\begin{aligned}
& \leqq\left(\sum_{|\gamma|<m-n / p}\left|\zeta_{\gamma}\right|+\sum_{|\gamma|<m-n / p}\left|\eta_{\gamma}\right|\right) \\
& \cdot\left\{1+\sum_{|\beta|=m}\left|\zeta_{\beta}\right|^{p-1}+\sum_{m-n / p \leqq|\beta|<m}\left|\zeta_{\beta}\right|^{(p-1)+c_{\beta \alpha}}+\sum_{m-n / p \leqq|\beta|<m}\left|\eta_{\beta}\right|^{(p-1)+c_{\beta \alpha}}\right\}
\end{aligned}
$$

where

$$
0 \leqq c_{\beta \alpha}<r_{\beta} \operatorname{Min}\left\{\frac{p-1}{p}+\frac{m-|\alpha|}{n}, 1\right\}-(p-1)
$$

and $r_{\beta}$ is any finite exponent such that

$$
r_{\beta}^{-1} \geqq p^{-1}-n^{-1}(m-|\beta|) .
$$

There exist exponents $r$ such that $r^{-1}>p^{-1}-n^{-1}$ and such that the form

$$
e(u ; v, w)=\sum_{|\alpha| \leqq m}\left\langle E_{\alpha}\left(x,\left\{D^{\gamma} u\right\},\left\{D^{\beta} v\right\}\right), D^{\alpha} w\right\rangle
$$

(where $E_{\alpha}\left(x,\left\{D^{\gamma} u\right\},\left\{D^{\beta} v\right\}\right)$ is the result of substituting $D^{\beta} u$ for $\eta_{\beta}, D^{\beta} v$ for $\zeta_{\beta}$ in $E(x, \eta, \zeta))$ is well defined for all $u \in W^{m-1, r}(\Omega)$ and $v$ and $w$ in $W^{m, p}(\Omega)$ and such that $e(u ; v, w)$ is continuous in the pair $(u, v)$ in $W^{m-1, r}(\Omega) \times W^{m, p}(\Omega)$ uniformly for $w$ in bounded sets of $w$ in $W^{m, p}(\Omega)$.

Let $b(u ; v, w)$ be a boundary form having the same property. Let $V$ be a closed subspace of $W^{m, p}(\Omega)$. Suppose that:

(a) For each $N>0$, there exists a continuous function $C_{N}(t)$ on $R^{1}, C_{N}(t)>0$ for $t>0, C_{N}(t) \rightarrow+\infty$ for $t \rightarrow+\infty$ such that

$$
\begin{gathered}
\operatorname{Re}\{e(u ; v, v-w)-e(u ; w, v-w)+b(u ; v, v-w)-b(u ; w, v-w)\} \\
\geqq C_{N}\left(\|v-w\|_{m, p}\right)\|v-w\|_{m, p}
\end{gathered}
$$

for all $u$ in $V$ with $\|u\|_{m-1, r} \leqq N$ and all $v$ and $w$ in $V$.

(b) There exists a continuous function $c(t)$ on $R^{1}$ with $c(t) \rightarrow+\infty$ as $t \rightarrow+\infty$ such that

$$
\operatorname{Re}\{e(u ; u, k u)+b(u ; u, k u)\} \geqq c\left(\|u\|_{m, p}\right)\|u\|_{m, p}
$$

for all $u \in V, k \geqq 1$.

Then for every $f$ in $V^{*}$, there exists $u$ in $V$ such that

for all $v$ in $V$.

$$
a(u, v)+b(u ; u, v)=(f, v)
$$


We omit the proof of Theorem 3, which is essentially the same as that of Theorem 1. We omit also the detailed statement of hypotheses on the boundary form $b(u ; v, w)$ based on the boundary case of the Sobolev Imbedding Theorem which would imply the continuity of $b(u ; v, w)$ in the sense required in the hypothesis of Theorem 3 .

This may be obtained by a direct analogue of the arguments of $\$ 3$ culminating in Proposition 3.2.

Let us conclude the present section with some brief remarks on the hypotheses (a) and (b) of Theorems 1 and 3 and the possibility of verifying them from assumptions on the Jacobi equations of the nonlinear system $A$.

Let $A_{\alpha, \beta}=\partial A_{\alpha} / \partial \zeta_{\beta}$. Then

$$
\begin{aligned}
& a(u ; v, v-w)-a(u ; w, v-w) \\
& =\sum_{|x| \leqq m,|\beta|=m} \int_{0}^{1}\left\langle A_{\alpha \beta}\left(x, u, \cdots, D^{m-1} u, t D^{m} v+(1-t) D^{m} w\right) D^{\beta}(v-w), D^{\alpha}(v-w)\right\rangle d t \\
& =\sum_{\alpha, \beta}\left\langle e_{\alpha \beta}(x, u, v, w) D^{\beta}(v-w), D^{\alpha}(v-w)\right\rangle .
\end{aligned}
$$

Thus estimates on $\operatorname{Re}\{a(u ; v, v-w)-a(u ; w, v-w)\}$ may be obtained from hypotheses on the form with coefficients $A_{\alpha \beta}$ of the type considered by Vishik in [23]. Similar considerations apply to the form $\operatorname{Re}\{a(u ; k u, u)\}$.

5. We turn now to the detailed consideration of the abstract theory of nonlinear functional equations involving monotone operators which underlies the results of $\$ 2$. For continuous operators in Hilbert space, the study of monotone operators was begun by G. J. Minty in [15] and extended by the writer in [3] and Minty in [16]. The extension to demi-continuous and hemi-continuous operators in Hilbert space was given by the writer in [4], [5], and [6]. The writer extended these results to separable reflexive Banach spaces in [7], as well as to classes of densely defined operators in [8] and [9]. A slightly later extension to reflexive Banach spaces without a separability assumption was given by Minty in [17]. (More special results in the Banach space context were obtained earlier by M. A. Krasnoselski [13] and Vainberg and Kachurovski [19].)

We propose in the present discussion to obtain a general theorem of this type in the most general context available, that of locally convex linear spaces.

Let $E_{1}$ and $E_{2}$ be two locally convex linear Hausdorff spaces over the real numbers. Let $\left(E_{1}, E_{2}\right)$ be a dual system in the sense of [11], i.e. for $u$ in $E_{1}$ and $v$ in $E_{2}$ we have a bilinear pairing $(u, v)$ defined such that:

(1) $(u, v)$ is continuous in $u$ on $E_{1}$ for fixed $v$ in $E_{2}$, and $(u, v)$ is continuous in $v$ on $E_{2}$ for fixed $u$ in $E_{1}$.

(2) If $v \in E_{2}, v \neq 0$, there exists $u$ in $E_{1}$ such that $(u, v) \neq 0$.

Let $T$ be a mapping (not necessarily linear) from $E_{1}$ to $E_{2} . T$ is said to be monotone if 


$$
(u-v, T u-T v) \geqq 0
$$

for all $u$ and $v$ in $E_{1} . T$ is said to be finitely continuous if it is continuous from every finite-dimensional subspace of $E_{1}$ to $E_{2}$.

Let $S$ be a subset of $E_{1} . K(S)$ denotes its closed convex hull in $E_{1}$. If $u \in K(S)-S$, then $S$ is said to envelop $u$ if for every finite-dimensional flat $F$ containing $u$, the boundary of $K(S) \cap F$ is contained in $S \cap F$.

If $C$ is a mapping of $E_{1}$ into $E_{2}$, it is said to be completely continuous with respect to $\left(E_{1}, E_{2}\right)$ if it is continuous and if the mapping $j$ of $E_{1}$ into $R^{1}$ given by $j(u)=(u, C u)$ is continuous on compact subsets of $E_{1}$.

THEOREM 4. Let $\left(E_{1}, E_{2}\right)$ be a dual system as above, $T$ a (not necessarily linear) mapping of $E_{1}$ into $E_{2}$ such that $T=T_{0}+C$ where $T_{0}$ is finitely continuous and monotone, $C$ is completely continuous with respect to $\left(E_{1}, E_{2}\right)$. Let $S$ be a subset of $E_{1}$ such that $K(S)$ is compact in $E_{1}$ and $u_{0}$ a point of $K(S)-S$ such that $S$ envelops $u_{0}$. Suppose that for given $w$ in $E_{2}$, we have

$$
\left(u-u_{0}, T u-w\right) \geqq 0
$$

for all $u$ in $S . T$ hen there exists $u_{1}$ in $K(S)$ such that $T u_{1}=w$.

The proof of Theorem 4 rests upon the two following lemmas.

LEMMA 5.1. Let $T_{0}$ be a finitely continuous map of $E_{1}$ into $E_{2}, u_{1} \in E_{1}$, $u_{2} \in E_{2}$. Suppose that

$$
\left(u-u_{1}, T_{0} u-u_{2}\right) \geqq 0
$$

for all $u$ in $E_{1}$. Then $T_{0} u_{1}=u_{2}$.

Proof of Lemma 5.1. Suppose $T_{0} u_{1} \neq u_{2}$. Then there exists $v$ in $E_{1}$ such that $\left(v, u_{2}-T_{0} u_{1}\right)>0$. For $t>0$, let $v_{t}=u_{1}+t v$ and substitute $v_{t}$ for $u$ in the inequality (5.2). We obtain

$$
t\left(v, T_{0} v_{t}-u_{2}\right) \geqq 0
$$

or cancelling the positive factor $t$,

$$
\left(v, T_{0} v_{t}-u_{2}\right) \geqq 0 .
$$

We rewrite this as

$$
\left(v, T_{0} v_{t}-T_{0} u_{1}\right) \geqq\left(v, u_{2}-T_{0} u_{1}\right)
$$

and let $t \rightarrow 0+$. Then $\left(v, T_{0} v_{t}-T_{0} u_{1}\right) \rightarrow 0$, and

$$
0 \geqq\left(v, u_{2}-T_{0} u_{1}\right)>0,
$$

a contradiction which implies that $T_{0} u_{1}=u_{2}$.

Q.E.D. 
LEMMA 5.2. Let $E_{1}$ be of finite dimension, $T$ a continuous map of $E_{1}$ into $E_{2}$ where $\left(E_{1}, E_{2}\right)$ is a dual system. Suppose $S$ is a bounded subset of $E_{1}$ which envelops 0. Suppose that

$$
(u, T u) \geqq 0
$$

for all $u$ in $S$. Then there exists $u$ in $K(S)$ such that $T u_{1}=0$.

Proof of Lemma 5.2. If $E_{1}$ is of finite dimension and $\left(E_{1}, E_{2}\right)$ is a dual system, $E_{2}$ is isomorphic to the dual space $E_{1}^{*}$ of $E_{1}$. Since the assertions of the lemma are invariant under the replacement of one topology on $E_{1}$ by an equivalent topology, we may assume that $E_{1}$ is a Hilbert space $H, E_{2}=E_{1}=H$, and the pairing is the inner product. Then $T$ maps $H$ into itself.

Let $T_{t}=(1-t) T+t I, 0 \leqq t \leqq 1$, and let $D$ be the interior of $K(S)$. Since the boundary of $K(S)$ is contained in $S, 0$ lies in $D$. We shall calculate the degree of $T_{t}$ on $D$ over 0 . For $t=1, T_{1}=I$ and this degree is 1 . On $\partial D$, the boundary of $D, T_{t} u \neq 0$ for $t>0$ since

$$
(u, T, u)=(1-t)(u, T u)+t\|u\|^{2} \geqq t\|u\|^{2}
$$

for $u \in \partial D \subset S$. If $T_{0}$ has a zero on $\partial D$, our proof is complete. Otherwise the degree of $T_{0}$ on $D$ over 0 equals the degree of $T_{1}$ on $D$ over 0 , which equals 1 . Hence there exists $u_{1}$ in $D$ such that $T_{0} u_{1}=T u_{1}=0$.

Q.E.D.

Proof of Theorem 4. We normalize the situation by replacing $u$ by $v=u-u_{0}$ and setting $T_{1} v=T\left(u-u_{0}\right)-w$. Then $S_{1}=\left\{v \mid v=u-u_{0}, u \in S\right\}$ surrounds 0 and the existence of a solution of $T u=w$ on $K(S)$ is equivalent to the existence of a solution of $T_{1} v=0$ on $K\left(S_{1}\right)$. Thus we may assume to begin with that $u_{0}=0$, $w=0$, and

$$
(u, T u) \geqq 0
$$

on $S$.

For fixed $u$ in $E_{1}$, let

$$
M_{u}=K(S) \cap\left\{v \mid\left(u-v, T_{0} u+C v\right) \geqq 0\right\} .
$$

The function

$$
\left(u-v, T_{0} u+C v\right)=\left(u, T_{0} u\right)-\left(v, T_{0} u\right)+(u, C v)-(v, C v)
$$

is continuous in $v$ on $K(S)$ for fixed $u$ in $E_{1}$. Hence $M_{u}$ is a closed subset of the compact set $K(S)$.

Let $\left\{u_{1}, \cdots, u_{r}\right\}$ be a finite set in $E_{1}, F_{0}$ the finite-dimensional subspace of $E_{1}$ generated by $\left\{u_{1}, \cdots, u_{r}\right\}$. By the Hahn-Banach Theorem, we can find a bounded idempotent mapping $P$ of $E_{1}$ into $E_{1}$ whose range is $F_{0}$ (i.e. $P$ is a projection on $\left.F_{0}\right)$. Let $P^{*}$ be the mapping of $E_{2}$ into itself given by

$$
\left(v, P^{*} w\right)=(P v, w)
$$


Then $P^{*}$ is well defined and is a bounded linear idempotent mapping of $E_{2}$. Let $F^{\prime}$ be the range of $P^{*}$. Then $\left(F_{0}, F^{\prime}\right)$ form a dual system. Let $R$ be the map of $F_{0}$ into $F^{\prime}$ given by $R u=P^{*} T u$. Since $T$ is finitely continuous and $F_{0}$ has finite dimension, $R$ is continuous. If $S_{0}=S \cap F_{0}, K\left(S_{0}\right)-S_{0}$ contains 0 and its boundary is contained in $S_{0}$ by hypothesis. For $u \in S_{0}$,

$$
(u, R u)=\left(u, P^{*} T u\right)=(P u, T u)=(u, T u) \geqq 0
$$

since $P u=u$ for $u \in F_{0}$. Applying Lemma 5.2, we find that there exists $v_{1}$ in $K\left(S_{0}\right) \subset K(S)$ such that

$$
P * T v_{1}=0
$$

For this element $v_{1}$, we have

$$
P^{*} T_{0} v_{1}=-P^{*} C v_{1},
$$

and for $u_{j}, 1 \leqq j \leqq r$,

Hence

$$
\begin{aligned}
0 & \leqq\left(u_{j}-v_{1}, T_{0} u_{j}-T_{0} v_{1}\right) \\
& =\left(u_{j}-v_{1}, T_{0} u_{j}\right)-\left(P\left(u_{j}-v_{1}\right), T_{0} v_{1}\right) \\
& =\left(u_{j}-v_{1}, T_{0} u_{j}\right)-\left(\left(u_{j}-v_{1}\right), P^{*} T_{0} v_{1}\right) \\
& =\left(u_{j}-v_{1}, T_{0} u_{j}\right)+\left(u_{j}-v_{1}, P^{*} C v_{1}\right) \\
& =\left(u_{j}-v_{1}, T_{0} u_{j}+C v_{1}\right) .
\end{aligned}
$$

$$
v_{1} \in \bigcap_{k=1}^{r} M_{u_{j}}
$$

i.e. the $\left\{M_{u}\right\}$ have the finite intersection property. By the compactness of $K(S)$ and the closedness of each $M_{u}$, it follows that $\bigcap_{u \in E_{1}} M_{u} \neq \varnothing$.

Let $u_{1}$ be an element of $\bigcap_{u \in E_{1}} M_{v}$. Then

$$
\left(u-u_{1}, T_{0} u+C u_{1}\right) \geqq 0
$$

for all $u$ in $E_{1}$. By Lemma 5.1, $T_{0} u_{1}=-C u_{1}$, i.e. $T u_{1}=0$.

Q.E.D.

As a specialization of Theorem 4 , we have the following:

Theorem 5. Suppose $X$ is a Banach space, $X^{*}$ its dual space, $T$ a mapping of $X^{*}$ into $X$ such that $T=T_{0}+C$ where $T_{0}$ is monotone, $T_{0}$ is continuous from finite-dimensional subspaces of $X^{*}$ to the weak topology of $X, C$ is continuous from the weak*-topology on bounded subsets of $X^{*}$ to the strong topology on $X$. Let $S$ be a bounded subset of $X^{*}$ which envelops $u_{0}, w$ an element of $X$ such that

$$
\left(u-u_{0}, T u-w\right) \geqq 0
$$

for all $u$ in $S$. Then there exists $u_{1}$ in $K(S)$ the weak*-closed convex hull of $S$ such that $T u_{1}=w$. 
Proof of Theorem 5. This is obtained from Theorem 4 by letting $E_{1}$ be $X^{*}$ with its weak*-topology, $E_{2}$ be $X$ with its weak topology.

We conclude our discussion with an extension of the result of Theorem 5 in a slightly different direction.

THeOREM 6. Let $X$ be a reflexive Banach space, $Y$ a Banach space, $X^{*}$ and $Y^{*}$ the dual spaces of $X$ and $Y$, respectively. Let $T$ be a mapping of $X$ into $Y^{*}$ such that $T=T_{0}+C$ where $T_{0}$ is finitely continuous and $C$ is continuous on bounded sets of $X$ from the weak topology of $X$ to the strong topology of $Y^{*}$. Suppose there exists a bounded linear operator $L$ from $X$ to $Y$ with dense range in $Y$ such that

$$
\left(L_{0} u-L_{0} v, T_{0} u-T_{0} v\right) \geqq 0
$$

for all $u$ and $v$ of $X$. Let $S$ be a bounded subset of $X$ which envelops a point $u_{0}$ of $K(S)$, the closed convex hull of $S$, and such that for a given $w$ in $Y^{*}$,

$$
\left(L u-L u_{0}, T u-w\right) \geqq 0
$$

for all $u$ in $S$. Then there exists $u_{1}$ in $K(S)$ such that $T u_{1}=w$.

Proof of Theorem 6. Let $L^{*}$ be the adjoint map to $L$ carrying $Y^{*}$ into $X^{*}$, $R=L^{*} T$. Then $R$ satisfies the hypotheses of Theorem 5 since inequality (5.3) is equivalent to

$$
(u-v, R u-R v) \geqq 0
$$

while inequality (5.4) is equivalent to

$$
\left(u-u_{0}, R u-L^{*} w\right) \geqq 0,
$$

and $L^{*}$ is a continuous linear mapping and hence weakly continuous. (The reflexivity of $X$ implies of course that $X=\left(X^{*}\right)^{*}$.) Theorem 5 implies that there exists $u_{1}$ in $K(S)$ such that

$$
L^{*} w=R u_{1}=L^{*} T u_{1} .
$$

Hence $L^{*}\left(T u_{1}-w\right)=0$. Since $L$ has dense range in $Y, L^{*}$ has a trivial null space. Thus $T u_{1}=w$.

Q.E.D.

A final remark: For complex linear spaces, the real inner product given by

$$
\operatorname{Re}(u, v)
$$

gives us the corresponding theorems in the complex form applied in the preceding sections. Finally, if $\operatorname{Re}(u, T u) \geqq C(\|u\|)\|u\|$ with $C(r) \rightarrow+\infty$ as $r \rightarrow+\infty$, then for every $w$ in $X^{*}, \operatorname{Re}(u, T u-w) \geqq\{C(\|u\|)-\|w\|\}\|u\| \geqq 0$ for $\|u\|$ sufficiently large.

\section{BIBLIOGRA PHY}

1. F. E. Browder, Functional analysis and partial differential equations. I, Math. Ann. 138 (1959), 55-79. 22-130.

2. — On the spectral theory of elliptic differential operators. I, Math. Ann. 142 (1961), 
3. - On the solvability of nonlinear functional equations, Duke Math. J. 30 (1963), $557-566$

4. - - Variational boundary value problems for quasi-linear elliptic equations of arbitrary order, Proc. Nat. Acad. Sci. U.S.A. 50 (1963), 31-37.

5. - Variational boundary value problems for quasi-linear elliptic equations. II, Proc. Nat. Acad. Sci. U.S.A. 50 (1963), 592-598.

6. - Variational boundary value problems for quasi-linear elliptic equations. III, Proc. Nat. Acad. Sci. U.S.A. 50 (1963), 794-798.

7. - - Nonlinear elliptic boundary value problems, Bull. Amer. Math. Soc. 69 (1963), 864-876

8. - Nonlinear equations of evolution, Math. Ann. 80 (1964), 485-523.

9. - Strongly nonlinear parabolic boundary value problems, Amer. J. Math. 86 (1964), 339-357.

10. - Nonlinear parabolic boundary problems of arbitrary order, Bull. Amer. Math. Soc. 69 (1963), 860-863.

11. G. Köthe, Topologische lineare Räume, Vol. 1, Die Grundlehren der mathematischen Wissenschaften in Einzeldarstellungen, Bd. 107, Springer, Berlin, 1960.

12. M. A. Krasnoselski, Topological methods in the theory of nonlinear integral equations, Gosudarstv. Izdat. Tehn.-Teor. Lit., Moscow, 1956. (Russian)

13. —_, On a new fixed point principle, Trudy Mat. Seminar Voronezh Univ. (1958), 87-90. (Russian)

14. J. Leray and J. Schauder, Topologie et équations fonctionnelles, Ann. Sci. École Norm. Sup. 51 (1934), 45-78.

15. G. J. Minty, Monotone (nonlinear) operators in Hilbert space, Duke Math. J. 29 (1962), 341-346.

16. - Two theorems on nonlinear functional equations in Hilbert space, Bull. Amer. Math. Soc. 69 (1963), 691-692.

17. __ On a "monotonicity" method for the solution of nonlinear equations in Banach spaces, Proc. Nat. Acad. Sci. U.S.A. 50 (1963), 1038-1041.

18. M. M. Vainberg, Variational methods for the study of nonlinear operators, Moscow, 1956. (Russian)

19. M. M. Vainberg and R. I. Kachurovski, On the variational theory of nonlinear operators and equations, Dokl. Akad. Nauk 129 (1959), 1199-1202. (Russian)

20. M. I. Vishik, Solution of a system of quasi-linear equations in divergence form under periodic boundary conditions, Dokl. Akad. Nauk 137 (1961), 502-505. Soviet Math. Dokl. 2 (1961), 293-297.

21. —_ Boundary value problems for quasi-linear strongly elliptic systems in divergence form, Dokl. Akad. Nauk SSSR 138 (1961), 518-521. Soviet Math. Dokl. 2 (1961), 643-647.

22. - Simultaneous quasi-linear equations with lower order terms, Dokl. Akad. Nauk SSSR 144 (1962), 13-16. Soviet Math. Dokl. 3 (1962), 629-633.

23. - Quasi-linear strongly elliptic systems of differential equations having divergence form, Trudy Moskov. Mat. Obšč. 12 (1963), 125-184. Trans. Moscow. Math. Soc. 1963, 140-208.

INSTITUTE FOR AdVANCED StUdy,

Princeton, NeW Jersey

University of Chicago,

Chicago, Illinois 